Hydrol. Earth Syst. Sci., 10, 277-287, 2006

www.hydrol-earth-syst-sci.net/10/277/2006/

(C) Author(s) 2006. This work is licensed

under a Creative Commons License.

\title{
Top-kriging - geostatistics on stream networks
}

\author{
J. O. Skøien, R. Merz, and G. Blöschl \\ Institute for Hydraulic and Water Resources Engineering, Vienna University of Technology, Vienna, Austria
}

Received: 29 September 2005 - Published in Hydrol. Earth Syst. Sci. Discuss.: 4 November 2005

Revised: 1 February 2006 - Accepted: 22 February 2006 - Published: 26 April 2006

\begin{abstract}
We present Top-kriging, or topological kriging, as a method for estimating streamflow-related variables in ungauged catchments. It takes both the area and the nested nature of catchments into account. The main appeal of the method is that it is a best linear unbiased estimator (BLUE) adapted for the case of stream networks without any additional assumptions. The concept is built on the work of Sauquet et al. (2000) and extends it in a number of ways. We test the method for the case of the specific 100-year flood for two Austrian regions. The method provides more plausible and, indeed, more accurate estimates than Ordinary Kriging. For the variable of interest, Top-kriging also provides estimates of the uncertainty. On the main stream the estimated uncertainties are smallest and they gradually increase as one moves towards the headwaters. The method as presented here is able to exploit the information contained in short records by accounting for the uncertainty of each gauge. We suggest that Top-kriging can be used for spatially interpolating a range of streamflow-related variables including mean annual discharge, flood characteristics, low flow characteristics, concentrations, turbidity and stream temperature.
\end{abstract}

\section{Introduction}

The Problem of Ungauged Basins (PUB) (Sivapalan et al., 2003) is one of the central problems in hydrology and related sciences. The problem consists of estimating streamflowrelated variables at locations where no measurements are available. Estimates can be obtained by a range of methods (e.g. Blöschl, 2005). A particularly appealing set of methods are geostatistics, which allow estimation of a variable including its uncertainty at locations where no measurements are available (Journel and Huijbregts, 1978). The main ad-

Correspondence to: J. O. Skøien

(skoien@hydro.tuwien.ac.at) vantage of geostatistical methods is that they are best linear unbiased estimators (BLUE); best meaning that the mean squared error is a minimum, linear meaning that the estimate is a weighted mean of the data in the area, and unbiased meaning that the mean expected error is zero (Journel and Huijbregts, 1978, p. 304). Geostatistical methods have evolved in the mining industry. The main problem consisted of estimating the expected ore grade (and its uncertainty) of a block using point samples of the ore grade in the area. To this end the spatial correlations of pairs of points are plotted versus their Euclidian distance. From this, the variogram is estimated which is then used to estimate the variable at the location of interest for a given block size from the point samples (Journel and Huijbregts, 1978).

The problem in catchment hydrology is quite different. The main difference is that catchments are organised into subcatchments. Unlike mining blocks, catchments are nested. Water follows a stream network. It is therefore clear that upstream and downstream catchments would have to be treated differently from neighbouring catchments that do not share a subcatchment. Therefore Euclidian distances between catchments are not the natural way of measuring the spatial distance of catchments. Estimation of variables on stream networks needs to use a topology that is different from the usual Euclidian topology.

Most applications of geostatistics to catchment hydrology, so far, have indeed used Euclidian distance between catchments, usually measured as the Euclidian distance between the gauges or the catchment centroids (e.g. Daviau et al., 2000; Adamowski and Bocci, 2001; Eaton et al., 2002; Skøien et al., 2003; Merz and Blöschl, 2005). Given the obvious nested structure of catchments it is surprising that very little research has been done on extending geostatistical concepts to catchments. There is one notable exception. Gottschalk and co-workers (Gottschalk, 1993a, b; Sauquet et al., 2000) have addressed this very issue. Gottschalk (1993a) first developed a method for calculating covariance along a

Published by Copernicus GmbH on behalf of the European Geosciences Union. 


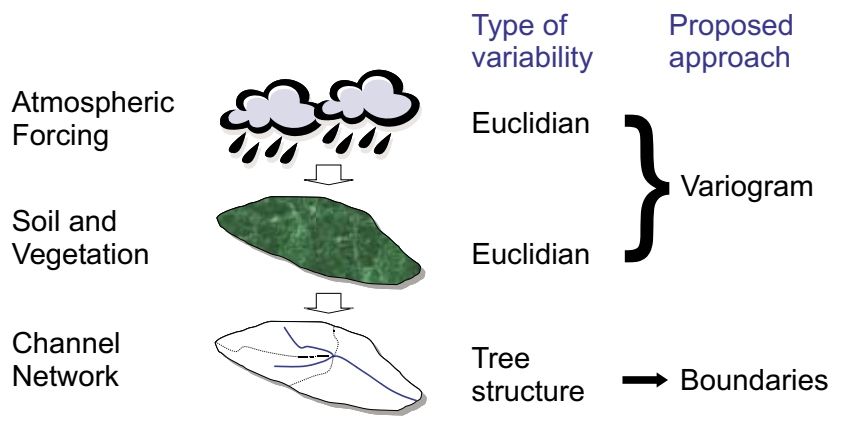

Fig. 1. Atmospheric forcing and soil and vegetation contribute to the runoff generation process locally and can be represented by point variograms. The channel network organises runoff into streams, which can be represented by the catchment boundaries.

river network and used this for interpolation along the network (Gottschalk, 1993b). Sauquet et al. (2000) further developed this method for mapping annual runoff along the river network using water balance constraints in the estimation procedure.

In this paper we propose a method of geostatistical estimation on stream networks that builds on the work of Sauquet et al. (2000). It extends the original work in a number of ways. First, we suggest that the interpolation method can be used, in an approximate way, for a range of streamflow-related variables including variables that are not fully mass conserving. Sauquet et al. (2000) interpolated mean annual runoff which is a mass conserving variable. Second, we use variograms while Sauquet et al. (2000) used covariances. This allows us to deal with variables that are non-stationary. Third, we account for local uncertainties of the measurements that may differ between locations. This allows us to exploit short records. Last, we illustrate the potential of the approach for estimating the uncertainty of the variable of interest in ungauged catchments.

In Sect. 2 we first review the basic concepts, and then present the methodology in detail. In Sect. 3 we illustrate the approach for the case of estimating the 100 year specific flood in ungauged catchments in Austria. This includes a comparison of the estimates with Ordinary Kriging as well as an analysis of the estimation uncertainties in ungauged catchments. Section 4 summarises the main implications for hydrological regionalisation.

\section{Method of Top-kriging}

\subsection{Concepts of Top-kriging}

There are two main groups of variables that control streamflow (Fig. 1). The first group consists of variables that are continuous in space. These variables include rainfall, evapotranspiration and soil characteristics. They are related to local runoff generation. In this context, runoff generation is conceptualised as a point process, i.e. runoff generation is assumed to exist at any point in the landscape. This concept is discussed in Woods and Sivapalan (1999). In a similar way, other streamflow-related variables can be conceptualised as continuous point processes on the local scale. For characterising these variables, Euclidian distances are appropriate. The spatial statistical characteristics of the point variables can be represented by the variogram (Skøien et al., 2003).

The second group of variables is related to routing in the stream network. These variables are affected by the catchment organisation of nested catchments where runoff accumulates along the stream network. Variables of this type include mean annual discharge, flood characteristics, low flow characteristics, concentrations, turbidity and stream temperature. These variables are only defined for points on the stream network. They cannot be represented by Euclidian distances. Rather they need to be represented by methods that reflect the tree structure of the stream network.

We propose a method that combines these two groups of variables in a geostatistical framework. We term the method topological kriging or Top-kriging, as it takes into account the topology of stream networks and nested catchments. The continuous process in space defined for point variables is represented by a variogram. The channel network structure and the similarity between upstream and downstream neighbours are represented by the catchment area that drains to a particular location on the stream network. The catchment areas are defined by their boundaries in space.

\subsection{Kriging basics}

In Euclidian kriging methods (such as Ordinary Kriging), the variable of interest is represented as a random field of values $z(\mathbf{x})$. Spatial similarity is represented by the variance between pairs of points as a function of their Euclidian distance. Kriging is then the best linear unbiased estimator, i.e. an interpolation method where the expected bias is zero and the expected kriging error is minimised. An unknown value $\hat{z}\left(x_{0}\right)$ of the variable at position $\boldsymbol{x}_{0}$ (i.e. the target position) can be estimated as a weighted average of the variable measured in the neighbourhood:

$\hat{z}\left(\boldsymbol{x}_{0}\right)=\sum_{i=1}^{n} \lambda_{i} z\left(\boldsymbol{x}_{i}\right)$

$\lambda_{i}$ is the interpolation weight of the measurement at position $\boldsymbol{x}_{i}$ and $n$ is the number of neighbouring measurements used for interpolation. The weights $\lambda_{i}$ can be found by solving the kriging system:

$$
\begin{aligned}
\sum_{j=1}^{n} \lambda_{j} \gamma_{i j}-\lambda_{i} \sigma_{i}^{2}+\mu & =\gamma_{0 i} \quad i=1, \ldots, n \\
\sum_{j=1}^{n} \lambda_{j} & =1
\end{aligned}
$$

The $\gamma_{i j}$ refers to the gamma value or the expected semivariance between two measurements $i$ and $j$, as found from a 
theoretical semivariogram model. $\mu$ is the Lagrange parameter. $\sigma_{i}^{2}$ represents the measurement error or uncertainty of measurement $i$. The use of measurement errors in the kriging equations is termed kriging with uncertain data (KUD) (de Marsily, 1986, p. 300; Merz and Blöschl, 2005).

\subsection{Interpolation and regularisation over catchment bound- aries}

In Top-kriging, the measurements are not point values but are defined over a non-zero catchment area $A$. In geostatistical terminology, $A$ is the support. A point variable $z(\boldsymbol{x})$ can be averaged over an area $A$ as:

$\bar{z}(A)=\frac{1}{A} \int_{A} w(\boldsymbol{x}) z(\boldsymbol{x}) d \boldsymbol{x}$

where $\bar{z}$ is the spatially averaged variable and $w(\boldsymbol{x})$ is a weighting function. If there is reason to assume the variable is conservative, or approximately conservative, as we do in this paper, the aggregation is linear, and $w(\boldsymbol{x})=1$. If a nonzero support $A$ is accounted for, the kriging system remains the same, but the gamma values between the measurements need to be obtained by regularisation (Cressie, 1991, p. 66). Assuming the existence of a point variogram $\gamma_{p}$, the gamma value or the semivariance between two measurements with catchment areas $A_{1}$ and $A_{2}$, respectively, is:

$$
\begin{aligned}
& \gamma_{12}=0.5 \times \operatorname{Var}\left(z\left(A_{1}\right)-z\left(A_{2}\right)\right) \\
& =\frac{1}{A_{1} A_{2}} \iint_{A_{1}} \gamma_{A_{2}}\left(\left|\boldsymbol{x}_{1}-\boldsymbol{x}_{2}\right|\right) d \boldsymbol{x}_{1} d \boldsymbol{x}_{2} \\
& -0.5 \times\left[\frac{1}{A_{1}^{2}} \iint_{A_{1}} \gamma_{A_{1}}\left(\left|\boldsymbol{x}_{1}-\boldsymbol{x}_{2}\right|\right) d \boldsymbol{x}_{1} d \boldsymbol{x}_{2}\right. \\
& \left.+\frac{1}{A_{2}^{2}} \int_{A_{2}} \int_{A_{2}} \gamma_{p}\left(\left|\boldsymbol{x}_{1}-\boldsymbol{x}_{2}\right|\right) d \boldsymbol{x}_{1} d \boldsymbol{x}_{2}\right]
\end{aligned}
$$

$\boldsymbol{x}_{1}$ and $\boldsymbol{x}_{2}$ are position vectors within each catchment used for the integration. The first part of this expression integrates all the variance between the two catchments, while the second part subtracts the averaged variance within the catchments. The second part is the smoothing effect of the support, which indicates that the variance of the averaged variable decreases as the support area increases. Equation (4) can be used to estimate the variogram of the averaged variable from the point variogram. This procedure is termed regularisation although most textbooks use the term only for the case when $A_{1}=A_{2}$. The gamma values are inserted into the kriging matrix Eq. (2) and the kriging system can be solved in the normal way to calculate the weights $\lambda_{i}$ for the interpolation scheme. The important thing in Top-kriging is that the integration is performed over the catchment area that drains to a particular location on the stream network. The location on the stream network is the outlet of the target catchment. It is hence possible to perform geo-statistics on stream networks.

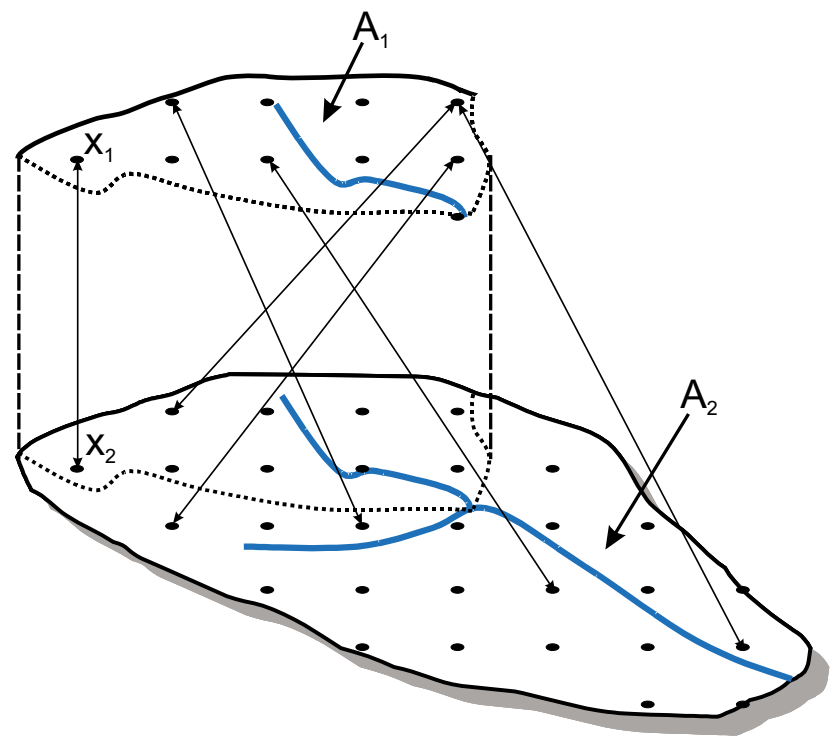

Fig. 2. Schematic stream network and catchment boundaries with point pairs shown.

The integration in Eq. (4) will, in most cases, be either very complex or impossible to carry out analytically. The integrals have to be replaced by sums and the catchment area is discretised by a grid. It is important to note that the grid has to be the same for each catchment every time it is discretised (Isaaks and Srivastava, 1989, p. 326). In fact, even slight differences of the grids or randomly chosen points will cause numerical problems that are likely to flaw the results as test simulations with the examples shown later have indicated. Figure 2 shows a schematic of two nested catchments, their discretisation by a square grid, and the distances between the discretised points within the catchments.

Many variables are likely to have a nugget effect that represents variability at scales much smaller than the distance between measurements. In the variogram the nugget appears as a discontinuity close to the origin. A point variogram with nugget variance $C_{0 p}$ can be expressed as:

$\gamma_{p}(h)=\left\{\begin{array}{c}0 \quad \text { if } h=0 \\ f(h)+C_{0 p} \text { otherwise }\end{array}\right.$

where $h$ is spatial distance and $f(h)$ is the variogram without nugget. If we regularise Eq. (5) with Eq. (4) directly, the nugget will vanish even for small catchments. We therefore propose to regularise the nugget separately. The nugget variance can be seen as the variance of a spatially independent random variable. Following Journel and Huijbregts (1978, p. 154-156), the regularised nugget variance for two catchments of different size $C_{0}\left(A_{1}, A_{2}\right)$, overlapping or not, can be generalised as:

$$
C_{0}\left(A_{1}, A_{2}\right)=0.5\left(\frac{C_{0 p}}{A_{1}}+\frac{C_{0 p}}{A_{2}}-\frac{2 C_{0 p} \cdot \operatorname{Meas}\left(\mathrm{A}_{1} \cap A_{2}\right)}{A_{1} A_{2}}\right)
$$



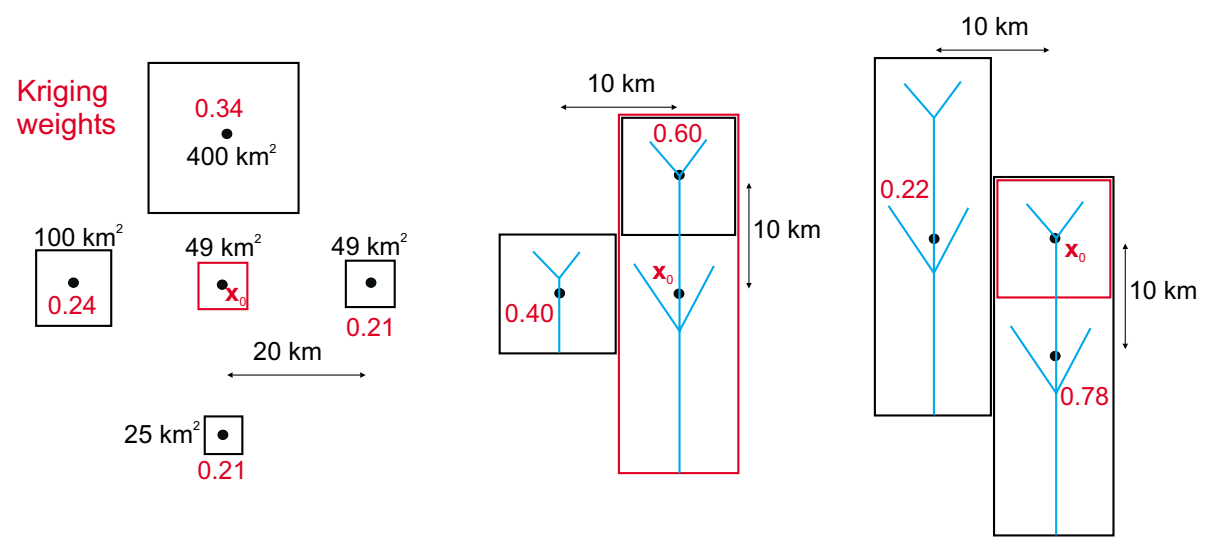

Fig. 3. Effect of catchment size (left) and nesting (centre and right) on the kriging weights $\lambda_{i}$ (red numbers) as estimated by Top-kriging. $\boldsymbol{x}_{0}$ indicates the centre of the target catchment.

where Meas $\left(\mathrm{A}_{1} \cap A_{2}\right)$ represents the area shared by the two catchments with areas $A_{1}$ and $A_{2}$. If the catchments are nested this will be $\min \left(A_{1}, A_{2}\right)$, if they are not this will be zero. The regularised nugget effect is then added to the regularised variogram of Eq. (4).

An advantage of kriging over some other interpolation methods is that it provides an estimate of the kriging variance of the estimate at any location. The kriging variance $\sigma_{R}^{2}$ represents the uncertainty of the estimates and is given by:

$\sigma_{R}^{2}=\sum_{i=1}^{n} \lambda_{i} \gamma_{i 0}+\mu$

$\gamma_{i 0}$ is the gamma value between the target catchment and the neighbouring catchments.

Figure 3 illustrates the merits of Top-kriging over Euclidian kriging methods such as Ordinary Kriging. In all three examples, the neighbouring catchments have the same centre-to-centre distance to the target catchment $(20 \mathrm{~km}$ in the left panel, $10 \mathrm{~km}$ in the centre and right panels). In Ordinary Kriging this would imply that the same weights $\lambda_{i}$ are assigned to all the neighbouring catchments ( 0.25 in the case of four neighbours, 0.5 in the case of two neighbours). In Top-kriging the weights are different. The example on the left shows the catchment size effect. The largest catchment has the largest weight, because this is regarded as the most certain, or having the least biased measurement in comparison to the mean. Although the $49 \mathrm{~km}^{2}$ catchment on the right is larger than the $25 \mathrm{~km}^{2}$ catchment at the bottom, their weights are similar which is because the $49 \mathrm{~km}^{2}$ catchment is closer to the $400 \mathrm{~km}^{2}$ catchment. This is because kriging reduces the weights of clustered samples in contrast to, e.g., inverse distance methods (Isaaks and Srivastava, 1989, pp. 318-321).

The centre and right panels in Fig. 3 show the effect of nesting. The sizes of the two neighbouring catchments are the same in each case $\left(100 \mathrm{~km}^{2}\right.$ in the centre panel, $300 \mathrm{~km}^{2}$ in the right panel). The centre panel indicates that the catchment that forms a subcatchment of the target catchment gets a larger weight even though size and distance are the same as those of the other catchment. The right panel shows the reverse case. Although the neighbours have the same areas and the same centre-to-centre distances to the target catchment, more weight is attached to the catchment into which the target catchment drains.

The weights have been obtained by the variogram shown later in this paper with the measurement errors set to zero. The relative effects of size and nesting would be similar for other variograms.

\section{Example application}

\subsection{Data}

The concept of Top-kriging is applied to an Austrian data set of 7000 catchments in this paper. For all of these catchments the stream network and the catchment boundaries were available. About 600 of these catchments were gauged. For these flood data were used with record lengths ranging from 5 to 110 years, most of them from 10 to 50 years. From these data, the specific 100 year flood, $Q_{100}$, was estimated using a Gumbel distribution. To be able to compare smaller and larger catchments, the $Q_{100}$ values were normalised to a catchment size of $100 \mathrm{~km}^{2}$, following Merz and Blöschl (2005):

$Q_{100 N}=\left(A \cdot \alpha^{-1}\right)^{\beta} Q_{100}$

where $A$ is the catchment area, $\alpha$ is the catchment area used for normalisation $\left(100 \mathrm{~km}^{2}\right)$ and $\beta$ was set to -0.33 . Although the kriging theory does not require the data to be normally distributed it is an advantage to reduce skewness. Because of this, the $Q_{100 N}$ values were logarithmically transformed before using them for interpolation:

$z=\ln \left(Q_{100 N}\right)$ 
The expected variance of the estimate of a Gumbel distribution is (e.g. Plate, 1993, p. 418):

$\sigma_{Q T}^{2}=\frac{s_{Q}^{2}}{n}\left[1+1.1396 K_{T}+1.100 K_{T}^{2}\right]$

where $T$ refers to the return period of the flood, $s_{Q}^{2}$ is the variance of the annual flood series, $n$ is the number of annual floods in the series and $K_{T}$ is a constant dependent on the return period of the estimate:

$K_{T}=-\frac{\sqrt{6}}{\pi}(\ln (-\ln (1-1 / T))+0.5772)$

$T=100$ in the present case. The variance was also logarithmically transformed by

$\sigma_{i}^{2}=\sigma_{z}^{2}=\ln \left(1+C V_{Q 100 N}^{2}\right)$

where the coefficient of variation is expressed as $C V_{Q 100 N}=\sigma_{Q 100 N} / \mu_{Q 100 N} . \quad \mu_{Q 100 N}$ is the mean of the data series. Before presentation, all values were back transformed.

The $Q_{100 N}$ values of the gauged catchments are referred to as measurements in this paper to illustrate the characteristics of Top-kriging. Their variances $\sigma_{Q 100}^{2}$ are referred to as measurement errors to illustrate how Top-kriging estimates uncertainties in ungauged catchments.

\subsection{Estimation of point variogram}

In order to apply Top-kriging a point variogram is needed which we back-calculated from the $\ln \left(Q_{100 N}\right)$ values at the gauged catchments. Kyriakidis (2004), Mockus (1998) and Skøien et al. (2003) provide methods for back-calculation. As there are too many stations for using the variogram cloud for fitting, we estimated a sample variogram with the pairs grouped into bins, similar to Matheron's (1965) traditional estimator, but with two more dimensions, the areas of the two catchments of a pair:

$\gamma_{\mathrm{obs}}\left(A_{1}, A_{2}, h\right)=\frac{1}{2 n\left(A_{1}, A_{2}, h\right)} \sum_{i=1}^{n\left(A_{1}, A_{2}, h\right)}\left[z\left(\boldsymbol{x}_{i}\right)-z\left(\boldsymbol{x}_{i}+\boldsymbol{h}\right)\right]^{2}$

where $h=|\boldsymbol{h}|$ is the distance between the centroids of the catchments, $n\left(A_{1}, A_{2}, h\right)$ is the number of catchment pairs with areas $A_{1}$ and $A_{2}$, and distance $h$ between the centroids. The bins were logarithmically distributed in all three dimensions. To reduce the number of bins and to increase the number of pairs in each bin, $A_{1}$ was always chosen as the smaller area of the two catchments in a pair

Following Skøien et al. (2003) a point variogram with a nugget effect of the following shape was assumed:

$\gamma_{p}(h)=a h^{b}\left(1-e^{-(h / c)^{d}}\right)+C_{0 p}$

$a, b, c$ and $d$ are parameters. The parameters can be interpreted as following: $a$ is related to the sill of the variogram, $c$

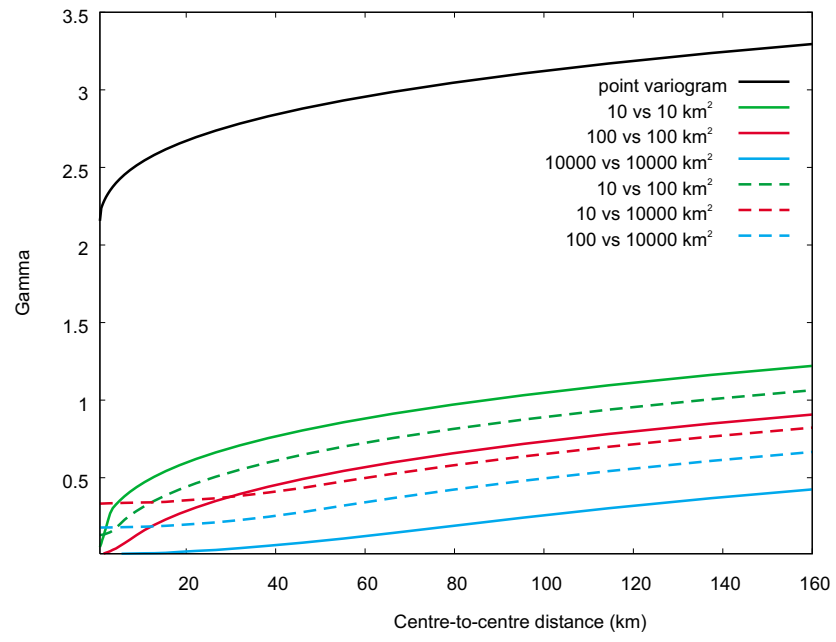

Fig. 4. Point variogram and regularised variograms of different catchment sizes.

is a correlation length, while $b$ and $d$ define the long and short distance slope of the variogram in a log-log plot, respectively. For a given bin, we calculated the regularised gamma values and nugget variance according to Eqs. (4) and (6) using average areas and distances within each bin and a square catchment area shape as an approximation. For each bin, we calculated a weighted relative difference between the observed gamma value $\gamma_{\text {obs }}$ and the regularised gamma value including nugget effect $\gamma_{\text {mod }}$, according to the Weighted Least Squares method of Cressie (1985):

$\operatorname{Err}\left(A_{1}, A_{2}, h\right)=n\left(A_{1}, A_{2}, h\right)\left(\frac{\gamma_{\text {obs }}\left(A_{1}, A_{2}, h\right)}{\gamma_{\bmod }\left(A_{1}, A_{2}, h\right)}-1\right)^{2}$

By minimising the sum of Err over all bins we found the parameters of the point variogram Eq. (14) as $a=2.99$, $b=0.0812, c=9690, d=0.2568, C_{0 p}=1.9668$.

From the point variogram we can now calculate a regularised variogram between two catchments as a function of their distance and catchment areas $A_{1}$ and $A_{2}$. Figure 4 shows the back-calculated point variogram together with a number of regularised variograms for different catchment areas, as examples. In all cases a square catchment shape was assumed. As the catchment area increases, the gamma values decrease because of the smoothing effect of regularisation. Catchments of different size will always have a variance between them, also when the centre-to-centre distance is zero. This is the reason why all variograms between catchments of different size start with an apparent nugget effect. The effect of the point nugget effect $C_{0 p}$ is dependent on the catchment size and degree of overlapping. There may be some uncertainty with estimating the point variogram as different point variograms can give similar regularised variograms. However, Top-kriging is not very sensitive to this as regularised variograms are used for catchment sizes that are of the same 


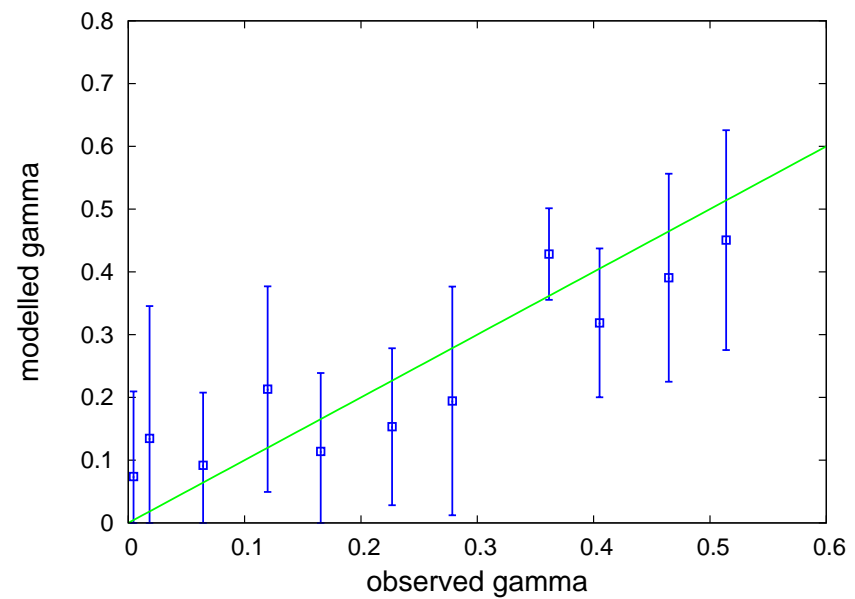

Fig. 5. Comparison between observed semivariances and mean and standard deviation (error bars) of modelled semivariances. Green line shows $\gamma_{\mathrm{obs}}=\gamma_{\text {mod }}$.

order of magnitude as those on which the observed variogram is based (Skøien and Blöschl, 2005b).

Figure 5 shows a comparison of the observed gamma values $\gamma_{\text {obs }}$ and the gamma values $\gamma_{\text {mod }}$ regularised from the point variogram. For the presentation, the observed gamma values were grouped and the mean and standard deviations of the modelled gamma values for each group are shown. The model has a tendency of overestimating the gamma values for small observed gamma values and the standard deviations of the modelled gamma values are relatively large. This is partly because the observed variogram Eq. (13) is more complex than the regularised version of Eq. (14) and partly because in some of the bins there are only few pairs. For the purpose of demonstrating the characteristics of Top-kriging, however, the fit was considered acceptable.

To assess the merit of Top-kriging relative to Ordinary Kriging we also interpolated the $Q_{100 N}$ values by Ordinary Kriging using a variogram of the type of Eq. (14) with the parameters $a=0.58, b=0.0010, c=29.8, d=0.667, C_{0 p}=0.0236$

\subsection{Results}

To understand and illustrate the characteristics of Topkriging this paper focuses on two sub-regions of the entire data set. We will discuss the estimates for individual catchments in a regional context for both regions. The first region is the river Mur and tributaries in Styria, southern Austria. In this region the focus is on the estimates on the tributaries. The second region is the river Inn and tributaries in Tyrol, western Austria, where the focus is on the main stream. In both examples, the Top-kriging estimates are compared with Ordinary Kriging estimates that use the Euclidian distance between the catchment centres to estimate the gamma values in the Kriging system.

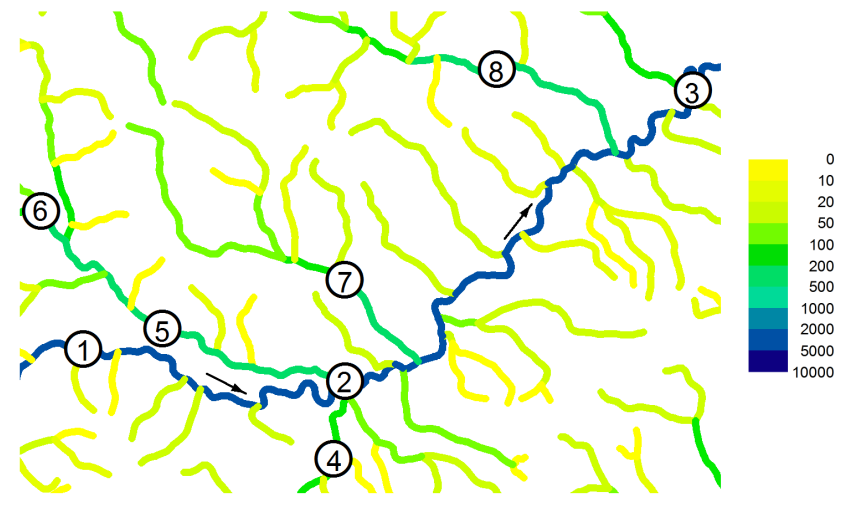

Fig. 6. Catchment area of the Mur and tributaries $\left(\mathrm{km}^{2}\right)$. Arrows show flow direction. Numbers refer to the stream gauges. Gauges 1-3 are situated directly on the Mur, the other gauges on the tributaries. The region represents a rectangle of 52 by $35 \mathrm{~km}$.

Figure 6 shows the catchment areas of the Mur and tributaries as well as the stream gauges. Stream gauges 1-3 are situated directly on the river Mur, the other gauges on the tributaries. The catchment area of the Mur almost doubles between stations 1 and 3 , from $2300 \mathrm{~km}^{2}$ to $4400 \mathrm{~km}^{2}$. There are four tributaries entering the river on this reach with catchment areas ranging from $200-500 \mathrm{~km}^{2}$.

Figure 7 presents the estimates of the normalised specific 100 year flood $Q_{100 N}$ from Top-kriging (top) and Ordinary Kriging (bottom) colour coded on the stream network. The measurements are shown as circles in both figures with the same colour coding. For both methods, the estimates next to the stream gauges are almost equal to the measurements of the stream gauge itself.

The Top-kriging estimates on the main river are similar to the measurements on the main river (gauges 1-3) and they do not change much along the reach. The estimates on the northern tributaries are much smaller than those on the main stream which is consistent with the measurements on the same tributaries (gauges 5 and 7). This is also reflected in the estimates for the other northern tributaries. On the southern side, the measurements are larger, so the Top-kriging estimates are generally much larger than those on the northern side of the Mur.

The Ordinary Kriging estimates differ substantially from the Top-kriging estimates. The main difference is that the estimates are not similar along the stream network as is the case of Top-kriging but similar along Euclidian distance in space. Although gauge 7 has measurements of 0.4 (red colour), most of the Ordinary Kriging estimates along this tributary are around 0.6 (yellow to green colours). This is because the estimates along this tributary are too much influenced by the measurements along the main river while they should be mainly influenced by the downstream gauge as is the case in Top-kriging. On the other hand, the estimates on the main stream are somewhat underestimated by Ordinary Kriging as 


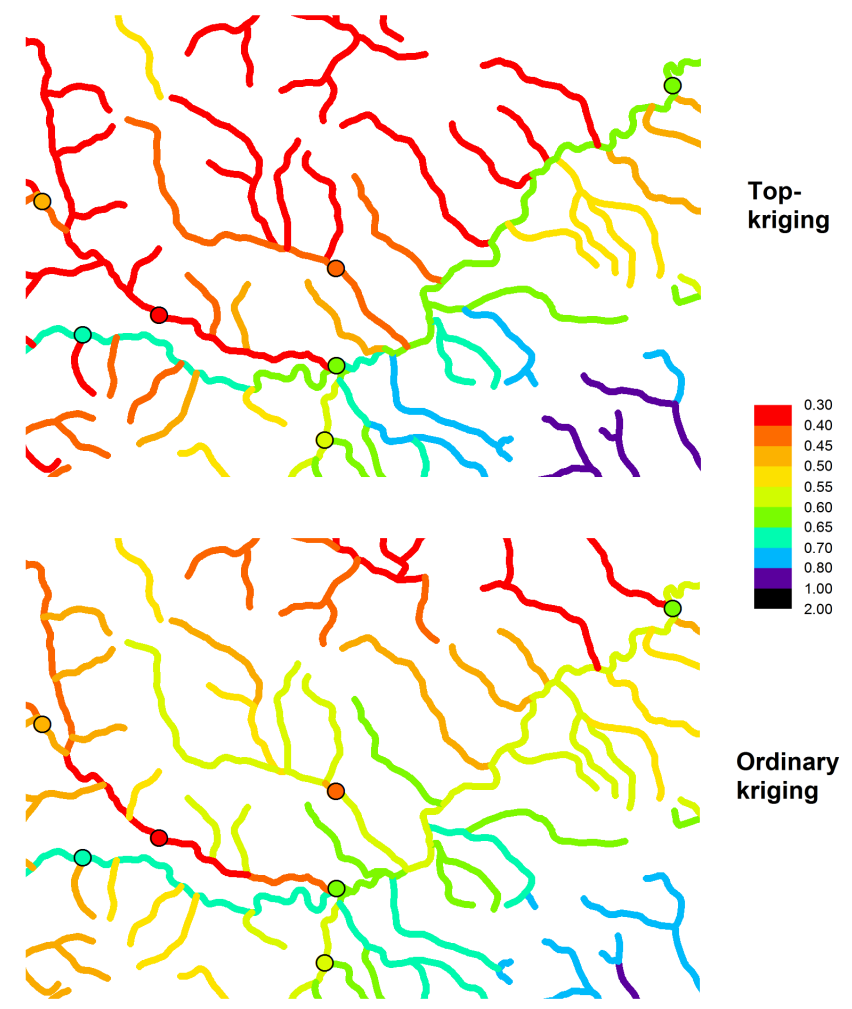

Fig. 7. Estimates of the normalised specific 100-year flood $Q_{100 N}$ from Top-kriging (top) and Ordinary Kriging (bottom) colour coded on the stream network of the Mur region. The measurements (i.e. values at the stream gauges) are shown as circles. Units are in $\mathrm{m}^{3} / \mathrm{s} / \mathrm{km}^{2}$.

they are too much affected by the measurements on the tributaries.

In order to examine the merits of Top-kriging more quantitatively we performed a cross validation procedure for both methods. A measurement was temporarily discarded from the sample data set and the $Q_{100 N}$ was then estimated by both Top-kriging and Ordinary Kriging for the same location from the remaining samples (Journel and Huijbregts, 1978, p. 352). The difference between the estimate so obtained and the measurement is a measure of the interpolation error. Figure 8 shows these differences for the two methods. The figure indicates that the estimates from Top-kriging are similar or better than the estimates from Ordinary Kriging in all cases but one (gauge 6). The Ordinary Kriging error for gauge 6 is smaller because it assigns more weights to gauges left of the region shown that possess larger $Q_{100 N}$ values. It is also worth noting that the errors are generally small for estimates on the main river (gauges 1-3), while they can be larger for the tributaries.

The kriging variances of the estimates in the Mur region, Eq. (7), are shown in Fig. 9 colour coded on the stream network. The kriging variances are expressed as the coefficient

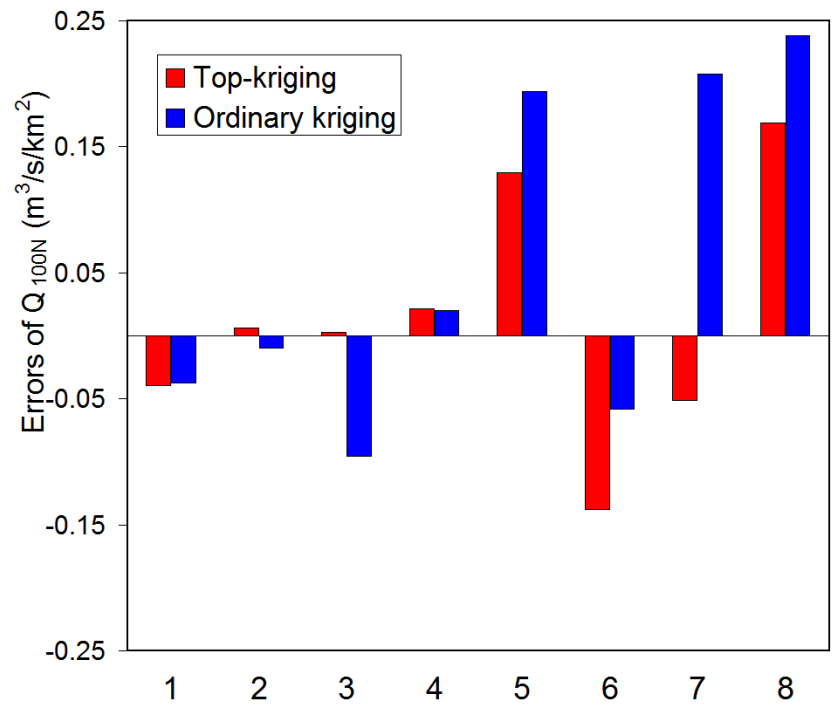

Fig. 8. Cross validation errors of $Q_{100 N}$ for the eight gauges in the Mur region. Station numbers as shown in Fig. 7.

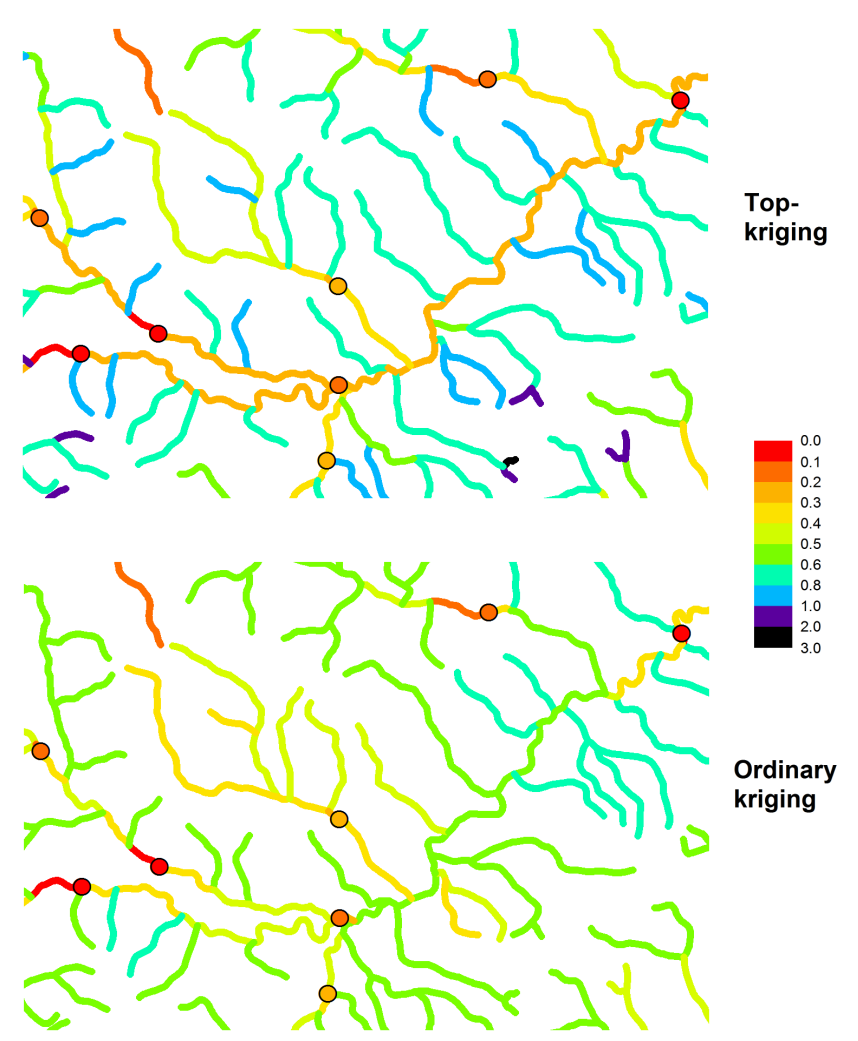

Fig. 9. Uncertainties of the normalised specific 100-year flood $Q_{100 N}$ from Top-kriging (top) and Ordinary Kriging (bottom), expressed as the coefficient of variation, colour coded on the stream network of the Mur region. Uncertainties of the measurements (i.e. values at the stream gauges) are shown as circles. 


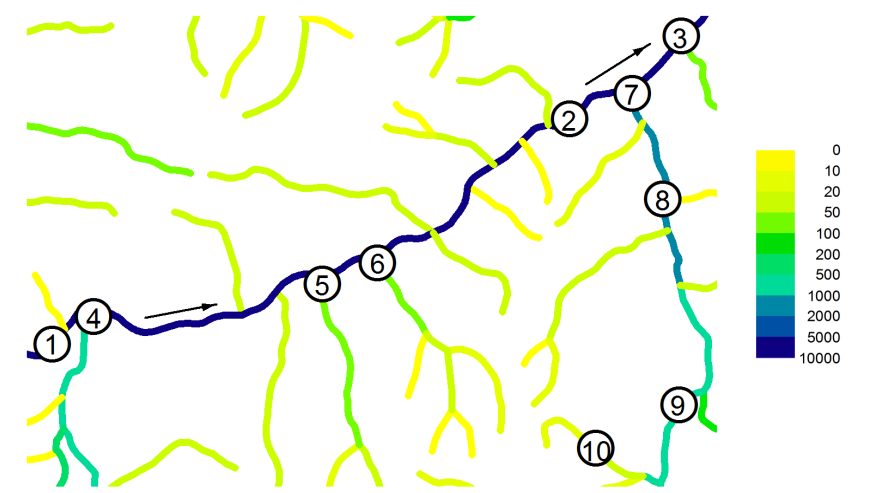

Fig. 10. Catchment area of the Inn and tributaries $\left(\mathrm{km}^{2}\right)$. Arrows show flow direction. Numbers refer to the stream gauges. Gauges 1-3 are situated directly on the Inn, the other gauges on the tributaries. The region represents a rectangle of 43 by $29 \mathrm{~km}$.

of variation $(\mathrm{CV})$ of the estimate:

$\mathrm{CV}=\frac{\sigma_{R}}{Q_{100 N}}$

The points represent the measurement error $\sigma_{i}^{2}$ at the stream gauges, again expressed as a coefficient of variation. The measurements have $\mathrm{CVs}$ in the range $0.05-0.3$. This is rather large, and is related to relatively short record lengths for some of the gauges. Both procedures estimate the lowest uncertainties close to the measurements, equal to or larger than the $\mathrm{CV}$ of the measurements. Note that the uncertainties on the stream network have been plotted for stream reaches of finite lengths as for these the catchment boundaries were available. The small uncertainties, strictly speaking, apply to the immediate neighbourhood of the gauges.

The uncertainties estimated by Top-kriging and Ordinary Kriging are very different for most of the stream network. Top-kriging (Fig. 9 top) gives relatively small uncertainties on the main river with CVs of around 0.2. This is only slightly larger than the $\mathrm{CV}$ of the measurements. On the other hand, the uncertainties of some of the tributaries are considerably larger. The uncertainties are small for those tributaries where measurements are available, but rather large for tributaries without any measurements. It is interesting that the uncertainty increases substantially with decreasing catchment area. For some of the smallest catchments, i.e. headwater catchments, CVs of more than 1 are estimated. These point to very uncertain estimates, which is not surprising as no measurements are near.

The uncertainties estimated by Ordinary Kriging (Fig. 9 bottom) contradict what one would intuitively expect. Most disturbing is that some of the smallest catchments have uncertainties equal to or smaller than the uncertainty of the main river. This is of course a result of the uncertainty being a function of Euclidian distance between catchment centre and measurements only, and not a function of the size and nesting of the catchments.

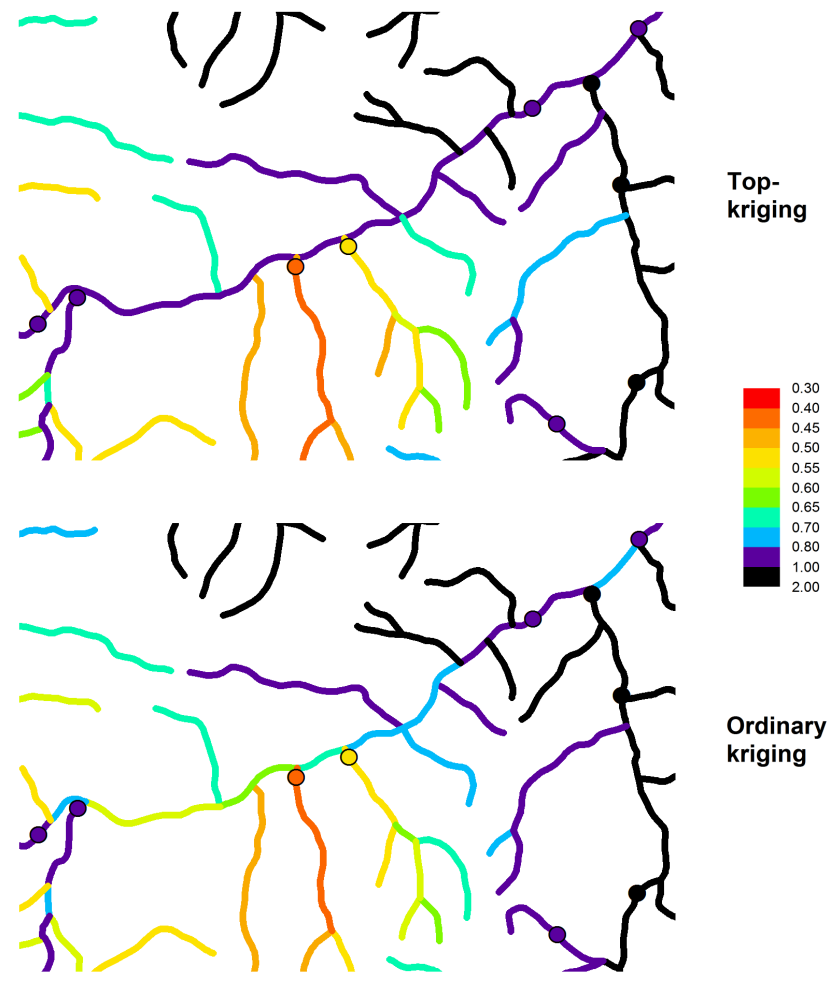

Fig. 11. Estimates of the normalised specific 100-year flood $Q_{100 N}$ from Top-kriging (top) and Ordinary Kriging (bottom) colour coded on the stream network of the Inn region. The measurements (i.e. values at the stream gauges) are shown as circles. Units are in $\mathrm{m}^{3} / \mathrm{s} / \mathrm{km}^{2}$.

The second example presented in this paper is the Inn region for which the catchment areas are shown in Fig. 10. Stream gauges 1-3 are situated directly on the river Inn, the other gauges on the tributaries. Between gauges 1 and 3 , the catchment area increases from $5800 \mathrm{~km}^{2}$ to $8500 \mathrm{~km}^{2}$. There are only two tributaries with an area of more than $100 \mathrm{~km}^{2}$ entering the Inn within this reach, but they are larger than the tributaries of the Mur. The Sill is the tributary gauged by gauge 4 just before the junction with the Inn, and has an area of $850 \mathrm{~km}^{2}$. The Ziller is the tributary gauged by gauges $7-10$, and has an area of $1135 \mathrm{~km}^{2}$.

The Top-kriging and Ordinary Kriging estimates of the normalised specific 100-year flood $Q_{100 N}$ are shown in Fig. 11 top and bottom, respectively. The $Q_{100 N}$ measured at the Sill (gauge 4) is similar to that measured at the Inn at gauge 1. The $Q_{100 N}$ measured at the Ziller (gauge 7) is larger than that measured at the Inn at gauge 1 but the $Q_{100 N}$ at the smaller tributaries (gauges 5 and 6 ) are much smaller than those of the Inn. Although the measurements on the tributaries show large variations, the Top-kriging estimates on the Inn change very little, which is consistent with the measurements.

Similar to the Mur case, the Ordinary Kriging estimates deviate considerably from Top-kriging but they deviate in a 


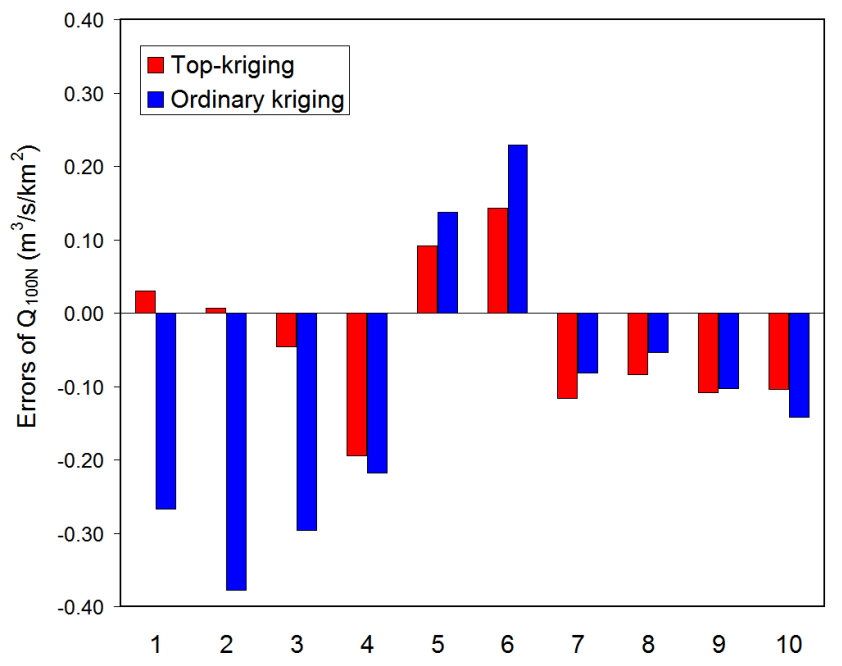

Fig. 12. Cross validation errors of $Q_{100 N}$ for the eight gauges in the Inn region. Station numbers as shown in Fig. 7.

different way because of different gauge locations. For the Mur case, the main differences were the estimates of the tributaries, which were too much affected by the main river. In the Inn case (Fig. 11), the estimates on the tributaries are similar to those of Top-kriging, but the estimates on the main river are obviously too much influenced by measurements on tributaries. The tributaries for which the centres are close to the centre of the Inn lie outside the region shown in Fig. 11 and have small measured $Q_{100 N}$ similar to the southern tributaries in Fig. 11.

The cross validation procedure confirms more quantitatively that the interpolation errors of Top-kriging are much smaller than those of Ordinary Kriging (Fig. 12). The difference between the two methods is largest along the main river (gauges 1-3). This is because estimates from Ordinary Kriging have their largest errors along the main river, while Top-kriging only gives small errors on the main river. The difference is smaller along the tributaries, but Top-kriging does give smaller errors than Ordinary Kriging for the majority of the gauges.

The estimated uncertainties of the estimates in the Inn region are shown in Fig. 13. Similar to Fig. 9, Fig. 13 shows that the uncertainties next to the stations are equal to the measurement uncertainties. The Top-kriging uncertainties along the main river and along tributaries with measurements are lower than the uncertainties of tributaries without measurements as would be expected. The uncertainties generally increase with decreasing catchment size. For the tributaries that are gauged close to the confluence with the main river (gauges 5 and 6) the uncertainty gradually increases as one moves away from the gauge towards the headwaters. However, if the tributary is gauged (e.g. by gauges 5 and 6 ) the estimates of the headwater catchments are less uncertain than the headwaters of ungauged tributaries (e.g. the tributaries

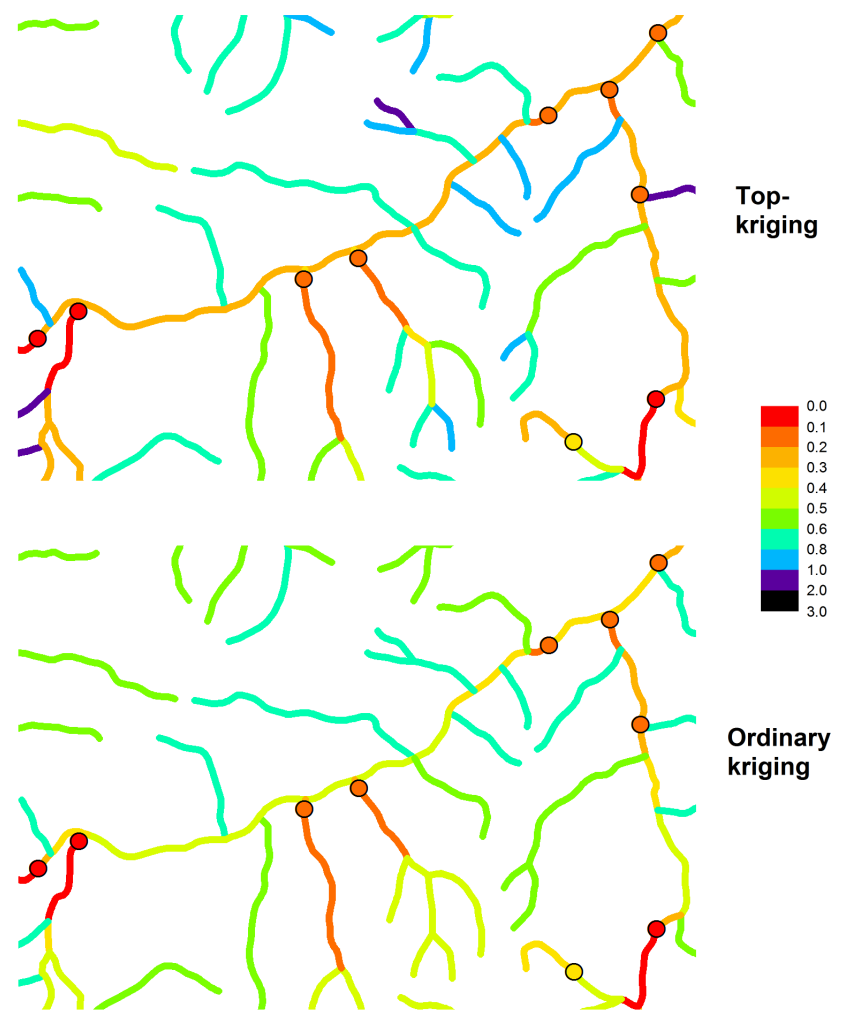

Fig. 13. Uncertainties of the normalised specific 100-year flood $Q_{100 N}$ from Top-kriging (top) and Ordinary Kriging (bottom), expressed as the coefficient of variation, colour coded on the stream network of the Inn region. Uncertainties of the measurements (i.e. values at the stream gauges) are shown as circles.

close to stream gauge 2). Overall, the Ordinary Kriging uncertainties indicate that the Euclidian distances do not reflect the intuitive distribution of estimation errors. The uncertainties are far too uniform within the region as they do not take into account the amount of information shared by gauged and ungauged catchments. In contrast, Top-kriging captures exactly this information, as expected.

\section{Conclusions}

We have presented Top-kriging as a spatial estimation method for streamflow-related variables. It takes both the area and the nested nature of catchments into account. The main appeal of the method is that it is a best linear unbiased estimator (BLUE) adapted for the case of stream networks without any additional assumptions. Because of the minimum number of assumptions we believe Top-kriging is the most natural method of estimating streamflow-related variables on stream networks.

The method provides more plausible and, indeed, more accurate estimates of the specific 100-year flood than Ordinary Kriging in the regions examined here. In the example 
of the Mur region we showed how the estimates of the tributaries are improved over Ordinary Kriging. In the example of the Inn region we showed how the estimates on the main stream are improved. In general, both the estimates on the main stream and the tributaries will be superior to Ordinary Kriging and the relative magnitude of the improvement will depend on the location of the gauges. In essence, Top-kriging treats upstream and downstream catchments differently from neighbouring catchments that do not share a subcatchment. This is also what one would do in manual interpolation. Typically, the effect of nesting will differ for upstream and downstream catchments. Larger catchments are generally given larger weights than smaller catchments (Fig. 3) and they are also likely to be well correlated with other large neighbours. It is therefore more likely that the nesting will have more impact on the weights of an upstream catchment than on those of a downstream catchment.

In addition to accounting for nested catchments, the method as presented here is able to exploit the information contained in short records (Eqs. 2 and 10). Variables estimated from short records are less certain than those from longer records. By allowing the measurement error to vary between gauges it was possible to use both short and long records and attribute more confidence to the longer records.

Top-kriging not only provides estimates of the variable of interest in ungauged catchments but also provides estimates of their uncertainty. The uncertainty patterns estimated by Top-kriging are very different from those of Ordinary Kriging. While the Ordinary Kriging uncertainty only depends on the centroid distances of gauged and ungauged catchments, the Top-kriging uncertainties fully take into account the nested nature of catchments. Figures 9 and 13 are typical of the way Top-kriging estimates interpolation uncertainty. On the main stream, where most of the gauges are, the estimated uncertainties are smallest. On tributaries that are gauged close to their confluence with the main river, the uncertainty gradually increases as one moves away from the gauge towards the headwaters. If a tributary is gauged, the estimates of the headwater catchments are less uncertain than the estimates of headwater catchments of similar size without a downstream gauge on the tributary. Locations between gauges at large rivers can also be considered as ungauged. However, it is in the headwater catchments where most of the uncertainty resides. The IAHS Decade on Predictions in Ungauged Basins (PUB) (Sivapalan et al., 2003) has predictive uncertainty of hydrological variables as its main focus. This is exactly what Top-kriging provides for the most natural case of best linear unbiased estimators.

Top-kriging assumes linear aggregation as it is a linear estimator. This means that, strictly speaking, the method only applies to variables that are mass conserving over nested catchments. We suggest that the method can also be profitably used, as an approximation, for variables that do not aggregate linearly but show a degree of averaging. The example of the specific 100-year flood shown here is not mass conserving and it was demonstrated that the Top-kriging estimates are much better than the Ordinary Kriging estimates. In fact, although Top-kriging is based on linear aggregation it does not necessarily reproduce the mass-balance of the variable of interest (Sauquet et al., 2000). This is consistent with our suggestion of the approximate use of Top-kriging for a range of streamflow-related variables. Such variables of interest in hydrology include mean annual discharge, flood characteristics, low flow characteristics, concentrations, turbidity and stream temperature.

Top-kriging as presented in this paper is based on a simple linear aggregation scheme. There are numerous opportunities for extending this simple scheme and still retaining the merits of a regionalisation procedure that naturally takes into account the area and nested structure of catchments. There are more complex ways of representing the logarithmic backtransformation of the variable of interest (e.g. Clark, 1998; Krige, 2004) and these could be used in a Top-kriging context. We have in a different context extended Top-kriging to a more complex scheme that takes auxiliary variables into account (such as mean annual precipitation and a lake index) to improve the estimates beyond simple interpolation. This is being used to estimate T-year floods for $26000 \mathrm{~km}$ of Austrian streams, which will be used for hazard zone planning in a project known as HORA. We are also planning to extend Top-kriging to space-time aggregation. Initial tests of space-time aggregation have been made in Skøien and Blöschl (2005b) and Skøien (2005a). Results of the more complex schemes will be reported in the near future.

Acknowledgements. The ideas of Top-kriging have evolved over the past ten years. Early discussions with M. Sivapalan and R. Grayson and more recent discussions with L. Gottschalk, I. Krasovskaia, E. Leblois and E. Sauquet have significantly contributed to our own ideas. The Austrian Academy of Sciences, project HÖ 18 provided financial support, the Austrian Hydrographic Service (HZB) provided the hydrographic data and J. Fürst provided the stream network data set. We are grateful for all of these contributions. We would also like to thank Gregor Laaha and an anonymous reviewer for their valuable comments.

Edited by: L. Pfister

\section{References}

Adamowski, K. and Bocci, C.: Geostatistical regional trend detection in river flow data, Hydrol. Processes, 15, 3331-3341, 2001.

Blöschl, G.: Rainfall-runoff modelling of ungauged catchments, Article 133, in: Encyclopedia of Hydrological Sciences, edited by: Anderson, M. G., pp. 2061-2080, Wiley, Chicester, 2005.

Clark, I.: Geostatistical estimation and the lognormal distribution, Geocongress, Pretoria, RSA, available at: http://uk.geocities. com/drisobelclark/resume/papers/Geocongress1998.zip (verified: 15 Sep 2005), 1998.

Cressie, N.: Fitting variogram models by weighted least squares, Math. Geol., 17, 563-586, 1985. 
Cressie, N.: Statistics for spatial data, Wiley, New York, NY, 1991.

Daviau, J.-L., Adamowski, K., and Patry, G. G.: Regional flood frequency analysis using GIS, L-moment and geostatistical methods, Hydrol. Processes, 14, 2731-2753, 2000.

de Marsily, G.: Quantitative hydrogeology, Academic Press Inc., London, 1986.

Eaton, B., Church, M., and Ham, D.: Scaling and regionalization of flood flows in British Columbia, Canada, Hydrol. Processes, 16, 3245-3263, 2002.

Gottschalk, L.: Correlation and covariance of runoff, Stochastic hydrology and hydraulics, 7, 85-101, 1993a.

Gottschalk, L.: Interpolation of runoff applying objective methods, Stoch. Hydrol. Hydraul., 7, 269-281, 1993 b.

Isaaks, E. H. and Srivastava, R. M.: An introduction to applied geostatistics, Oxford University Press, New York, 1989.

Journel, A. G. and Huijbregts, C. J.: Mining geostatistics, Academic Press, London, UK, 1978.

Krige, D. G.: Some practical aspects of the use of lognormal models for confidence limits and block distributions in South African gold mines, Journal of The South African Institute of Mining and Metallurgy, 104, 285-289, 2004.

Kyriakidis, P. C.: A geostatistical framework for area-to-point spatial interpolation, Geographical Analysis, 36, 259-289, 2004.

Matheron, G.: Les variables regionalisées et leur estimation, Masson, Paris, France, 1965.

Merz, R. and Blöschl, G.: Flood frequency regionalisation - Spatial proximity vs. catchment attributes, J. Hydrol., 302, 283-306, 2005.

Mockus, A.: Estimating dependencies from spatial averages, J. Computational Graphical Stat., 7, 501-513, 1998.
Plate, E. J.: Statistik und angewandte Wahrscheinlichkeitslehre für Bauingenieure, Ernst \& Sohn Verlag für Architektur und technische Wissenschaften, Berlin, 1993.

Sauquet, E., Gottschalk, L., and Leblois, E.: Mapping average annual runoff: a hierarchical approach applying a stochastic interpolation scheme, Hydrol. Sci. J., 45, 799-815, 2000.

Sivapalan, M., Takeuchi, K., Franks, S. W., Gupta, V. K., Karambiri, H., Lakshmi, V., Liang, X., McDonnell, J. J., Mendiondo, E. M., O'Connell, P. E., Oki, T., Pomeroy, J. W., Schertzer, D., Uhlenbrook, S., and Zehe, E.: IAHS Decade on Predictions in Ungauged Basins (PUB), 2003-2012: Shaping an exciting future for the hydrological sciences, Hydrol. Sci. J., 48, 857-880, 2003.

Skøien, J. O. and Blöschl, G.: Geostatistical interpolation of runoff, General Assembly 2005 of European Geosciences Union, Poster EGU05-A-06526, Vienna, Austria, 2005a.

Skøien, J. O. and Blöschl, G.: Spatio-temporal geostatistical analyses of runoff and precipitation, in: Geostatistics for Environmental Applications, Proceedings of the fifth European conference on Geostatistics for Environmental Applications, Neuchâtel, Switzerland, 2005b.

Skøien, J. O., Blöschl, G., and Western, A. W.: Characteristic space-time scales in hydrology, Water Resour. Res., 39, 1304, 2003.

Woods, R. and Sivapalan, M.: A synthesis of space-time variability in storm response: Rainfall, runoff generation, and routing, Water Resour. Res., 35, 2469-2485, 1999. 